\title{
Performance of Inductive Coupled Power Transfer Versus the Coil Shape - Investigation using Finite Element Analysis
}

\author{
Mohd Fakhizan Romlie ${ }^{1, ~ *}$, Kevin Lau ${ }^{1}$, Mohd Zaifulrizal Zainol ${ }^{1,2}$, Mohd Faris Abdullah ${ }^{1}$, \\ and Ramani Kannan ${ }^{1}$ \\ ${ }^{1}$ Universiti Teknologi PETRONAS, 32610 Seri Iskandar, Perak, Malaysia. \\ ${ }^{2}$ Universiti Kuala Lumpur Malaysian Institute of Marine Engineering Technology (UniKL MIMET), \\ Jalan Pantai Remis, 32200 Lumut, Perak, Malaysia.
}

\begin{abstract}
The objective of this paper is to investigate the impact of the spiral coil shape of inductive coupled power transfer on its performance. The coil shapes evaluated are: circular, square and pentagon spiral shapes. The coils are modelled in Ansoft Maxwell software. Simulations are carried out to determine the mutual inductance, coupling coefficient and magnetic flux density. The performance in term of magnetic flux density, mutual inductance and coupling coefficient of the three coils shapes are compared. Of the three shapes, the pentagon is shown to have the best performance in term of its mutual inductance, coupling coefficient and magnetic flux density.
\end{abstract}

\section{Introduction}

Wireless Power Transfer (WPT) is a technology widely researched nowadays. This is due to the fact that the conventional wired system is messy, inconvenience and also may cause hazards such as electric shock or electrocution [1]. Meanwhile, the WPT is convenience, not messy and can help to achieve cleaner and greener energy options [2]. The technology is researched for various application such as mobile devices charging [3], electric vehicle charging [4], implantable medical devices and even for huge power transmission i.e transmitting electrical power without transmission lines [6].

Two most common WPT system are Inductive Coupled Power Transfer (ICPT) and Capacitive Coupled Power Transfer (CCPT) [7]-[10]. ICPT system uses two closely spaced coils, one primary or transmitter and the other one secondary or receiver. The current flowing through the primary coil generates a magnetic flux that is received by the secondary coil. Capacitive coupling uses two parallel plate-pairs, separated by a gap: The energy fed to the receiver plate is dependent on the electric field in both plate pairs. Generally, ICPT operates based on magnetic field and CCPT is based on electric field. Figure 1 shows the overview of ICPT and CCPT.

This paper emphases on ICPT. The ICPT system comprises of two independent electrical systems with mutual coupling. The two coils or winding are physically separated by an air

* Corresponding author: mohdhazwan@ump.edu.my 
gap. Since the ICPT involves magnetic coupling, there is a need to understand the magnetic field characteristics of materials used in various shapes, parameters and conditions. This magnetic field characteristic will influence the mutual inductance, coupling coefficient and thus the performance of the ICPT.

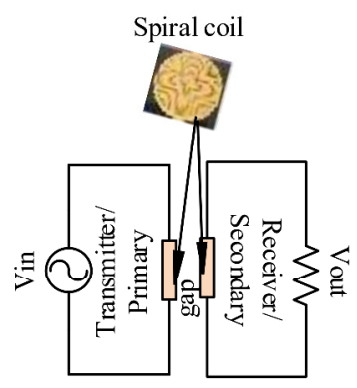

(a)

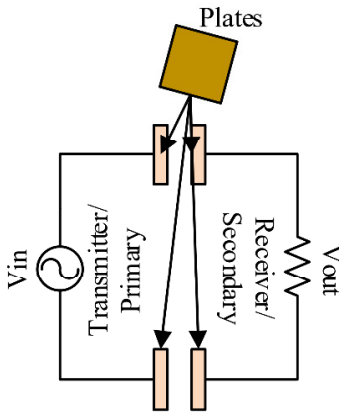

(b)

Fig. 1. (a) ICPT, (b) CCPT.

The shape and design of the coil play important role in ICPT. Different shape and design of the coil generate different patterns of the magnetic field and flux distribution. A shape may not generates a stable and suitable magnetic field and therefore affecting the efficiency of the system. For example, a research done by [11], shown that a spiral coil without any core have a better performance than a rounded winding of coil. It even have less resistance. In [12], it has shown that rectangular spiral coil can improve the power transfer capability as compared to circular and also having higher tolerance for misalignment.

Realizing the significance of the coil shapes on the ICPT performance, this paper aim to investigate the performance of ICPT for various shapes of the spiral coil. The shapes evaluated are circular, square and pentagon spiral coils. The performance is evaluated based on their magnetic flux density, mutual inductance and coupling coefficient. It is aim to understand further insight on the magnetic fields distributions in the coils and impact to the ICPT performance.

\section{Coupling coefficient and Mutual Inductance}

During the wireless power transmission between the primary coil and the secondary coil, there will be some leakage inductance. Meanwhile, mutual inductance is caused by the magnetic flux from primary coil cutting the secondary coil to induce the voltage and current in the secondary coil. Sometimes, the leakage inductance can be higher than mutual inductance in a loosely coupled system, which will reduce the magnetizing flux [1], [13]. The relationship between mutual inductance, $M$ and coupling coefficient, $k$ is given by

$$
M=k \sqrt{L_{1} L_{2}}
$$

where, $L_{1}$ is the inductance of primary coil and $L_{2}$ is the inductance of secondary coil. The power transferred to the receiver side, $P_{2}$ can be calculated by

$$
P_{2}=\omega M^{2} I_{P}^{2} / L_{2}
$$

where, $\omega$ is the frequency and $I_{p}$ is the current in primary coil. From this relationship, it is known that the output power also depends on mutual inductance. Higher mutual inductance means higher efficiency of the ICPT. Therefore, for the purpose of investigating the 
performance of ICPT for various shapes of the coil, this research observes the mutual inductance and coupling coefficient of the system.

There are many methods to calculate the mutual inductance such as Maxwell formulas, Grover's methods, Neumann's integrals and Finite Element Analysis (FEA) [14]. In this work, the FEA software, namely Ansoft Maxwell is used to determine the coupling coefficient and mutual inductance. The software is used to model the ICPT for all the shapes and simulation will be done in a 3D environment. The FEA is chosen because this method does not involve complex manual calculation and the software can promise consistent and reliable results for various shapes that will be evaluated.

\section{Methodology}

The comparative study of various coil shapes impact on the wireless power transfer performances is done by using FEA software, namely Ansoft Maxwell. The plane view of the coil design for the three shapes are shown in Figure 2. The 3-D design of the coil are shown in Figure 3, Figure 4 and Figure 5 for circular, square and pentagon spiral coils, respectively. The parameters for the design are summarized in Table 1 and Table 2.

The coils dimension, thickness, no of turns and separation between traces are given in Table 1 for the three shapes compared. As seen from the table the values are not exactly the same for all the shapes but are very close to each other. This is because it is impossible to have exactly the same values for the three different shapes. However, for this comparison, we are trying to achieve the same self-inductance for all the three shapes. Therefore, we have to vary other parameters accordingly. As shown in Table 2, the self-inductance for circular and pentagon are the same. However, the square has slightly higher value of the selfinductance. Note that the primary or transmitter and secondary or receiver coils are using exactly the same dimension and parameters values.

In this papers, the 3-D simulations were carries out to obtain the magnetic flux density for the shapes. Then, the mutual inductance and coupling coefficient at different air gap distance were also obtained using 3-D simulations. The simulations results are presented and discussed in the following section.

Table 1. The parameters for the coil shapes.

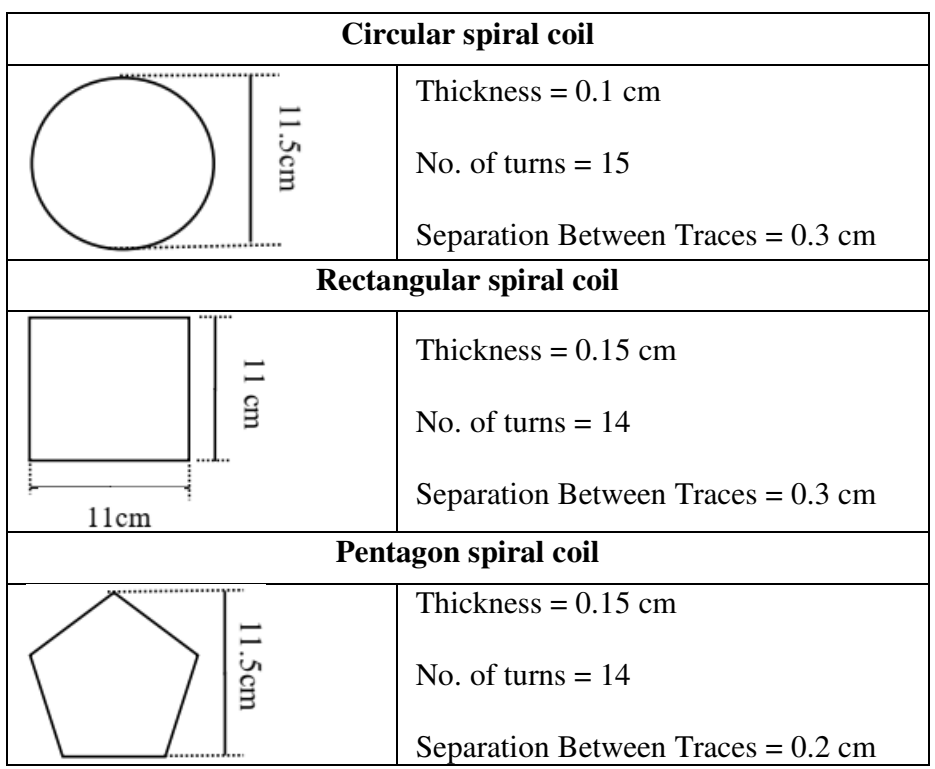


Table 2. Self-inductance of the coil (primary same as secondary side).

\begin{tabular}{|l|l|}
\hline Coil shapes & Inductance $(\mu \mathrm{H})$ \\
\hline Circular & $\mathrm{L} 1=10.1$ \\
\hline Square & $\mathrm{L} 1=10.7$ \\
\hline Pentagon & $\mathrm{L} 1=10.1$ \\
\hline
\end{tabular}

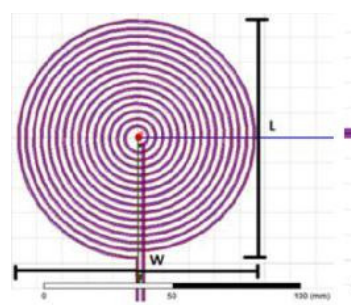

(a)

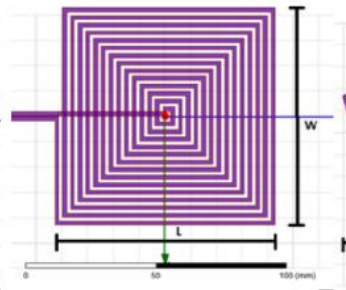

(b)

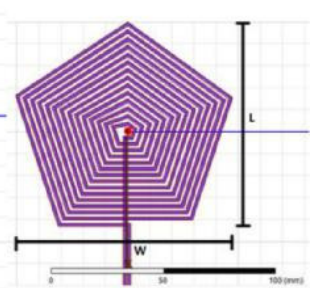

(c)

Fig. 2. Plane view of (a) circular, (b) square, (c) pentagon spiral coil design in Ansoft Maxwell.

Fig. 3. 3-D Circular spiral coil design in Ansoft Maxwell.
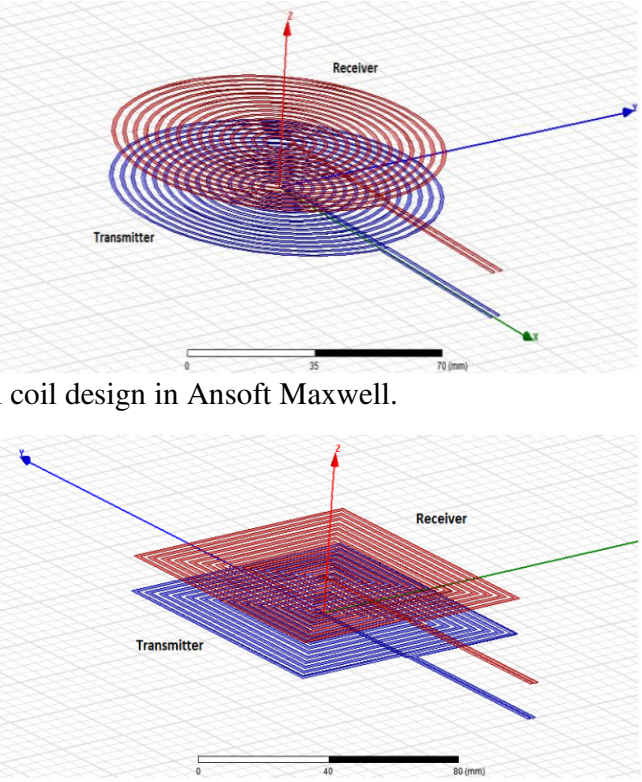

Fig. 4. 3-D Square spiral coil design in Ansoft Maxwell.

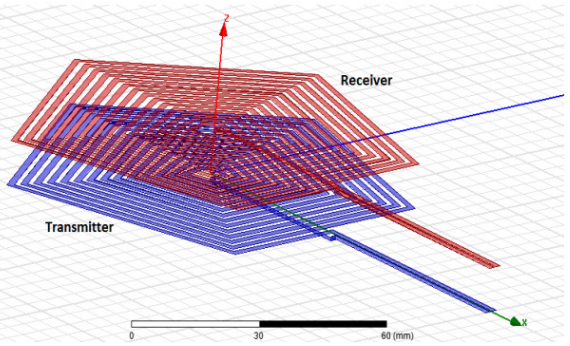

Fig. 5. 3-D Pentagon spiral coil design in Ansoft Maxwell. 


\section{Results and Discussion}

The magnetic flux density contour plots obtained from the FEA using the software are shown in Figure 6, Figure 7 and Figure 8 for circular, square and pentagon spiral coils, respectively. From Figure 6, for the circular spiral coil, the highest flux density is $4.7 \times 10^{-4} \mathrm{~T}$ and the lowest value is $3.37 \times 10^{-5} \mathrm{~T}$. It seems that the flux density is consistent throughout the coils where almost all the coils areas having the highest flux density value. Then, the value decreasing as it is getting further outside the coils areas. Meanwhile, from Figure 7, for the square spiral coil, the highest flux density is $5.13 \times 10^{-4} \mathrm{~T}$ and the lowest value is $3.77 \times 10^{-5} \mathrm{~T}$. Compared to the spiral planar coils, the values are higher. However, the flux distribution is not as consistent. For the pentagon spiral coil, the highest flux density is 5.60 $\mathrm{x} 10^{-4} \mathrm{~T}$ and the lowest value is $4.15 \times 10^{-5} \mathrm{~T}$ as seen in Figure 8. This is the highest amongst the three shapes. The flux distribution is more consistent than the planar coil but less than the spiral coil. Overall, the pentagon shape is having the most flux density and this is also reflected to the mutual inductance and coupling coefficient values as plotted in Figure 9 and Figure 10, respectively. For the same distance, the pentagon is having the highest mutual inductance and coupling coefficient followed by square and spiral shape. As the distance increased, the values are exponentially decaying. All the values are obtained from the FEA simulation software.

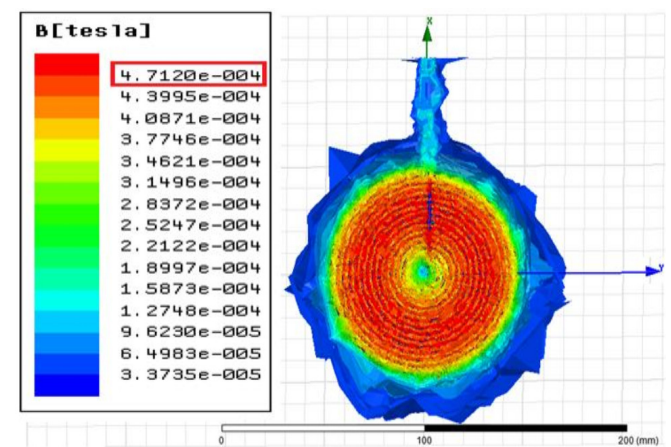

Fig. 6. Magnetic flux density of spiral planar coil.

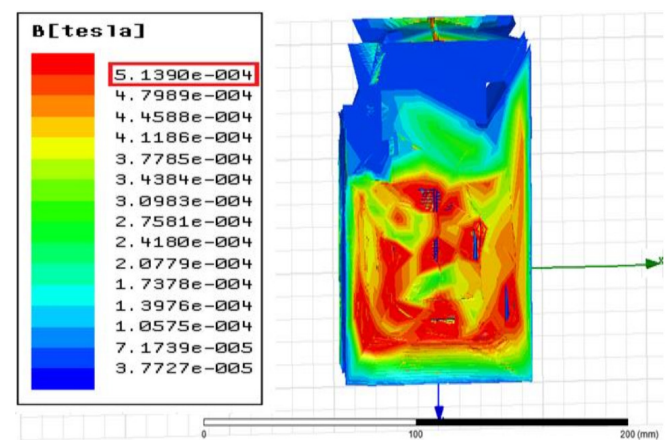

Fig. 7. Magnetic flux density of square planar coil. 

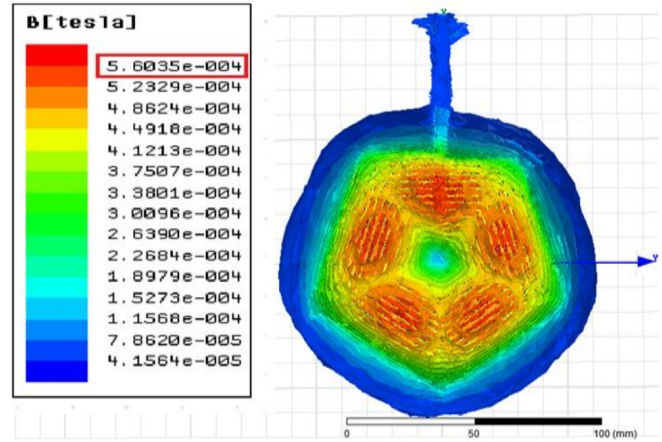

Fig. 8. Magnetic flux density of pentagon planar coil.

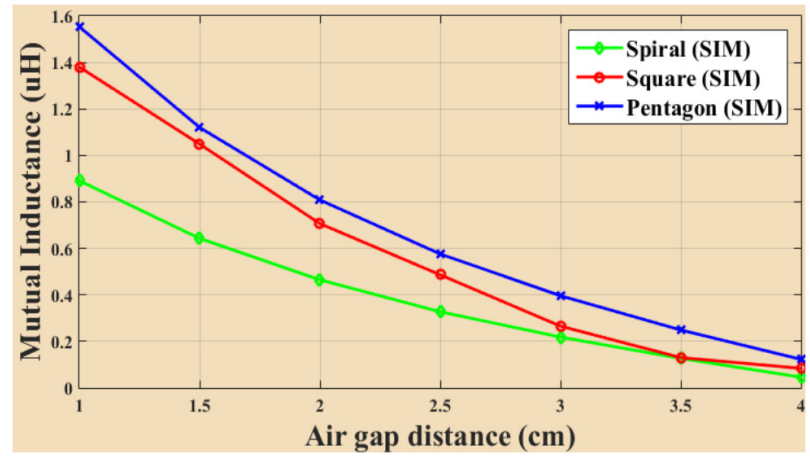

Fig. 9. Mutual inductance versus air gap distance.

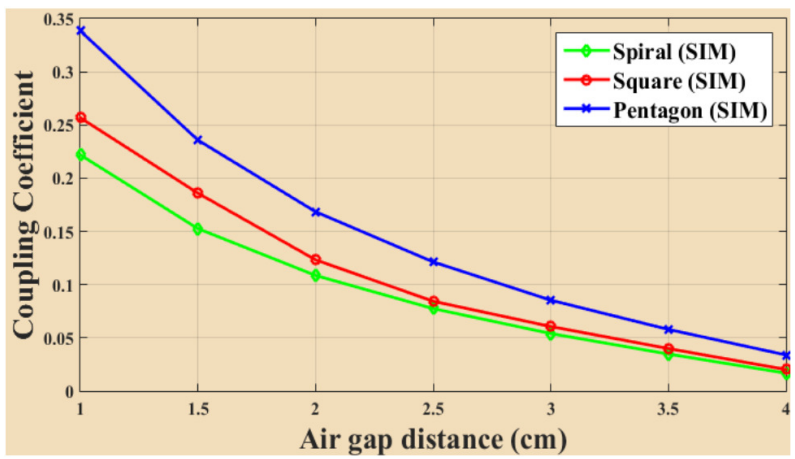

Fig. 10. Coupling coefficient versus air gap distance.

\section{Conclusion}

A comparative study of various shape of coils for ICPT has been investigated using FEA simulation. Three shape of coils were designed and simulated using Ansoft Maxwell to calculate the mutual inductance and coupling efficient over various air gap distance. Different coil shapes produce different magnetic flux. The flux contributes to the mutual inductance and coupling coefficient. The higher the magnetic flux, the higher the mutual inductance, and the higher the coupling coefficient. However, mutual inductance and coupling will decrease when the air gap distance increases. Comparing the performance of the three shapes, the pentagon spiral shape is the showing the best performance. Future work is needed to validate the simulation results experimentally. 
The authors would like to thank the Ministry of Higher Education (MOHE), Malaysia for providing the Fundamental Research Grant Scheme (FRGS) to perform this research and not forgetting UTP for their support.

\section{References}

1. A. J. L. Villa, J. Sallán, A. Llombart, and J. F. Sanz, Appl. Energy, 86 (3), pp. 355-363, (2009).

2. Y. Trivedi, 8 IEEE Commun. Mag. - Commun. Stand. Suppl., pp. 8-13, (2016).

3. L. Olvitz, D. Vinko, and T. Švedek, "Wireless Power Transfer for Mobile Phone Charging Device," in Proceedings of the 35th International Convention MIPRO, pp. 141-145 (2012).

4. K. A. Kalwar, M. Aamir, and S. Mekhilef, Renew. Sustain. Energy Rev., 47, pp. 462475, (2015).

5. X. Li, C. Tsui, and W. Ki, IEEE J. Solid-State Circuits, 50 (4), pp. 978-989, (2015).

6. S. Alexzander and I. K. Anbumalar, "Recent trends in power systems (wireless power transmission system) and supercapacitor application," in International Conference on Sustainable Energy and Intelligent Systems (SEISCON 2011), no. Seiscon, pp. 416-420 (2011).

7. A. I. Al-Kalbani, M. R. Yuce, and J. M. Redoute, IEEE Antennas Wirel. Propag. Lett., 13, pp. 1168-1171, (2014).

8. L. Huang and A. P. Hu, Electron. Lett., 51 (22), pp. 1806-1807, (2015).

9. a. W. Green, "10 kHz inductively coupled power transfer - concept and control," Proc. 5th Int. Conf. Power Electron. Var. Drives, pp. 694-699, (1994).

10. S. K. Mishra, R. Adda, A. K. Rathore, S. Sekhar, and A. Joshi, IET Power Electron., 9 (5), pp. 997-1008, (2016).

11. C. Fernández, O. García, R. Prieto, J. A. Cobos, S. Gabriels, and G. Van Der Borght, "Design Issues of a Core-less Transformer for a Contact-less Application," in Seventeenth Annual IEEE Applied Power Electronics Conference and Exposition APEC, pp. 339-345 (2002).

12. J. Sallan, J. L. Villa, a. Llombart, and J. F. Sanz, IEEE Trans. Ind. Electron., 56 (6), pp. 2140-2149, (2009).

13. K. Aditya and S. S. Williamson, Transp. Electrif. Conf. Expo (ITEC), 2014 IEEE, pp. $1-6,(2014)$.

14. O. H. Stielau and G. a Covic, "Design of loosely coupled inductive power transfer systems," Power Syst. Technol. 2000. Proceedings. PowerCon 2000. Int. Conf., pp. 8590 vol.1, (2000). 